\title{
Function of the nucleus of the solitary tract in the therapeutic effect of obesity medication lorcaserin
}

Obesity and overweight are leading healthcare issues in developed countries. Despite considerable clinical demand, limited pharmaceutical treatment options are currently available. Amongst those recently developed are the serotonin $2 \mathrm{C}$ receptor $(5-\mathrm{HT} 2 \mathrm{CR})$ agonist lorcaserin, glucagon-like peptide-1 receptor (GLP-1R) agonists (e.g. liraglutide) and melanocortin-4 (MC4R) agonists (e.g. setmelanotide). Defining whether these medications target the same or parallel brain circuits to improve obesity is the objective of the present work. 5-HT2CRs, GLP-1Rs, and MC4Rs are distributed throughout the brain including in the key homeostatic region of the nucleus of the solitary tract (NTS) ${ }^{(1,2)}$. The NTS has a major role in the regulation of food intake and several neuropeptides, including GLP-1 produced by preproglucagon (PPGNTS) neurons, are localized here ${ }^{(3)}$.

Adult male and female mice were used to investigate the therapeutic mechanism of obesity medications lorcaserin. To examine the potential interaction between the serotonin and GLP-1 circuits, neurons within the NTS making GLP-1 were ablated (PPG ${ }^{\mathrm{NTS}}$ ablation) or GLP-1 receptors were specifically knocked down within the NTS (GLP-1R ${ }^{\mathrm{NTS}}$ knockdown). All studies were conducted in accordance with The Animals (Scientific Procedures) Act 1986 and the principles of the 3Rs.

Our results demonstrate that $5-\mathrm{HT}_{2} \mathrm{R}$ agonist lorcaserin requires functional $\mathrm{PPG}^{\mathrm{NTS}}$ neurons to reduce food intake in mice. Lorcaserin significantly reduced cumulative $1-4$ hour food intake in control mice but not $P P G^{\text {NTS }}$ ablated mice $\left(F_{(1,26)}=9.977\right.$, $\mathrm{p}=0.004)$. Further analysis of the underlying neurocircuitry shows that approximately one-third of $\mathrm{PPG}^{\mathrm{NTS}}$ neurons express $5-\mathrm{HT}_{2 \mathrm{C}} \mathrm{Rs}(38.8 \pm 2.8 \%)$ and display the marker of neuronal activity c-FOS following lorcaserin treatment in mice. We next demonstrate, by knocking down expression of the GLP-1R within the NTS, that GLP-1R NTS neurons are necessary for 5-HT $2 \mathrm{C}$ dependent hypophagia. 1-4 hour cumulative food intake was significantly decreased in control mice but not GLP-1R knockdown mice following NTS 5- $\mathrm{HT}_{2 \mathrm{C}} \mathrm{R}$ activation $\left(\mathrm{F}_{(2,13)}=10.22, \mathrm{p}=0.002\right)$. We next established that $\mathrm{PPG}^{\mathrm{NTS}}$ neurons are also partly required for the anorectic effect of MC4R agonist melanotan-II (MT-II). MTII reduced cumulative food intake in both PPG ${ }^{\text {NTS }}$ neuron ablated and control mice at 1-4 hours (treatment $\mathrm{F}_{(1,11)}=107.5, \mathrm{p}<0.0001$ ). However, $\mathrm{PPG}_{\mathrm{NTS}}$ neuron ablated mice were resistant to the anorectic effect of MTII 21 hours (treatment $\mathrm{F}_{(1,11)}=4.733, \mathrm{p}=0.052$ ).

These data provide insight into the therapeutic mechanisms of obesity mediations and reveal key brain circuits modulating feeding.

Nutrition in the treatment, management and prevention of disease: nutrition strategies for disease prevention, nutrition in the management and treatment.

\section{Acknowledgments}

This work was supported by the BBSRC (BB/R01857X/1 and BB/N017838/1).

\section{References}

1. D'Agostino G, Lyons D, Cristiano C et al. (2018) Cell Metabolism 28(4), 533-534.

2. Alhadeff AL, Mergler BD, Zimmer DJ et al. (2017) Neuropsychopharmacology 42, 1471-1479.

3. Holt MK, Richards JE, Cook DR et al. (2019) Diabetes 68(1), 21-33. 